\title{
Statistical Heuristic Wettability Analysis of Randomly Textured Surfaces \\ Supporting Information
}

\author{
Md. Arifur Rahman Khandoker ${ }^{1}$, Kevin Golovin $^{1 *}$ \\ ${ }^{1}$ Okanagan Polymer Engineering Research \& Applications Laboratory, School of Engineering, University \\ of British Columbia, Kelowna, BC, V1V 1V7, Canada. \\ *Correspondence to: kevin.golovin@ubc.ca
}

\section{Table of contents}

Propagation of uncertainties from uncorrelated independent random variables................... S2

Derivation of the standard deviation for Wenzel contact angle, $\theta_{W}^{*} \ldots \ldots \ldots \ldots \ldots \ldots \ldots \ldots \ldots \ldots . \quad \mathrm{S} 2$

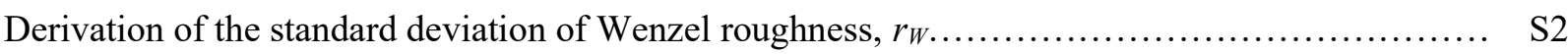

Derivation of the standard deviation for feature spacing ratio, $D^{*} \ldots \ldots \ldots \ldots \ldots \ldots \ldots \ldots \ldots \ldots \ldots . \quad \mathrm{S} 2$

Derivation of the standard deviation for statistical feature spacing ratio, $D_{\text {stat }}^{*} \ldots \ldots \ldots \ldots \ldots \ldots \ldots . \mathrm{S} 3$

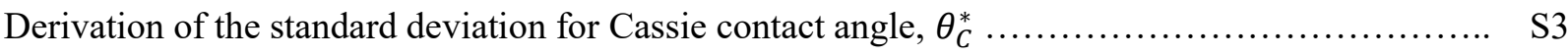

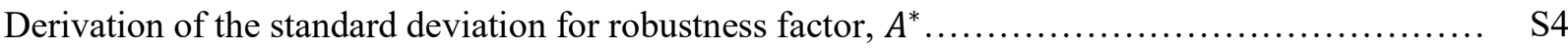

Derivation of the standard deviation for statistical robustness factor, $A_{\text {stat }}^{*} \ldots \ldots \ldots \ldots \ldots \ldots \ldots \ldots \ldots \ldots \ldots \ldots$

Derivation of the standard deviation for critical contact angle, $\theta_{\text {crit }} \ldots \ldots \ldots \ldots \ldots \ldots \ldots \ldots \ldots \ldots . \quad \mathrm{S} 4$

Derivation of the standard deviation for apparent contact angle in mixed Cassie state, $\theta_{M}^{*} \ldots \ldots \ldots . \quad$ S5

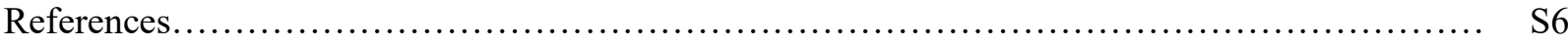




\section{Propagation of uncertainties from uncorrelated independent random variables}

If a random variable, $f(x, y, z)$, is a function of uncorrelated random variables, $x, y, z$, with random uncertainties (standard deviation of the mean) of $\sigma_{x}, \sigma_{y}, \sigma_{z}$, respectively, then the uncertainty in $f(x, y, z), \sigma_{f}$, is given by the quadrature sum of the uncertainties multiplied by a sensitivity factor as ${ }^{1}$,

$$
\sigma_{f}=\sqrt{\left(\frac{\partial f}{\partial x}\right)_{y, z}^{2} \sigma_{x}^{2}+\left(\frac{\partial f}{\partial y}\right)_{x, z}^{2} \sigma_{y}^{2}+\left(\frac{\partial f}{\partial z}\right)_{x, y}^{2} \sigma_{z}^{2}}
$$

This equation for independent error propagation in quadrature is used to derive equations for determining the standard deviations of Eq. (1) - (11), which are Eq. (S1) - (S9), taking the following assumptions:

1. Errors of the variables were independent.

2. Only the considered variables contribute to error.

3. All angles were in degrees $\left(^{\circ}\right)$.

\section{Derivation of the standard deviation for Wenzel contact angle, $\theta_{W}^{*}$}

Wenzel contact angle, $\theta_{W}^{*}$, From Eq. (1),

$$
\begin{aligned}
& \cos \theta_{W}^{*}=r \cos \theta \\
& \frac{\partial \theta_{W}^{*}}{\partial r}=\frac{-1}{\sqrt{1-(r \cos \theta)^{2}}} \cos \theta \\
& \frac{\partial \theta_{W}^{*}}{\partial \theta}=\frac{-1}{\sqrt{1-(r \cos \theta)^{2}}}(-r \sin \theta) \\
& d \theta_{W}^{*}=\frac{180^{\circ}}{\pi} \sqrt{\left(\frac{\partial \theta_{W}^{*}}{\partial r}\right)^{2} d r^{2}+\left(\frac{\partial \theta_{W}^{*}}{\partial \theta}\right)^{2} d\left(\frac{\pi \theta}{180^{\circ}}\right)^{2}} \\
& \Delta \theta_{W}^{*}=\frac{180^{\circ}}{\pi} \sqrt{\frac{(\cos \theta \Delta r)^{2}+\left(\frac{\pi}{180^{\circ}} r \sin \theta \Delta \theta\right)^{2}}{1-(r \cos \theta)^{2}}}
\end{aligned}
$$

\section{Derivation of the standard deviation of Wenzel roughness, $\boldsymbol{r}_{W}$}

Wenzel roughness, $r_{W}$, from Eq. (2),

$$
\begin{gathered}
r_{W}=1+0.01 S_{d r} \\
\Delta r_{W}=0.01 \Delta S_{d r}
\end{gathered}
$$

\section{Derivation of the standard deviation for feature spacing ratio, $D^{*}$}

Feature spacing ratio, $D^{*}$, from Eq. (3), 


$$
\begin{aligned}
& D^{*}=\left(\frac{D+R}{R}\right)^{2} \\
& d D^{*}=\sqrt{\left(\frac{\partial D^{*}}{\partial(D+R)}\right)^{2} d(D+R)^{2}+\left(\frac{\partial D^{*}}{\partial R}\right)^{2} d R^{2}} \\
& d D^{*}=2\left(\frac{D+R}{R}\right)^{2} \sqrt{\left(\frac{d(D+R)}{(D+R)}\right)^{2}+\left(\frac{d R}{R}\right)^{2}} \\
& \Delta D^{*}=2 D^{*} \sqrt{\left(\frac{\Delta(D+R)}{(D+R)}\right)^{2}+\left(\frac{\Delta R}{R}\right)^{2}}
\end{aligned}
$$

\section{Derivation of the standard deviation for statistical feature spacing ratio, $D_{\text {stat }}^{*}$}

Similarly, replacing $(D+R), R$ and $D^{*}$ in Eq. (S3) with $R S_{m}, S_{a l}$ and $D_{\text {stat }}^{*}$, the standard deviation of the statistical feature ratio, $D_{\text {stat }}^{*}$, can be found as,

$$
\Delta D_{\text {stat }}^{*}=2 D_{\text {stat }}^{*} \sqrt{\left(\frac{\Delta R S_{m}}{R S_{m}}\right)^{2}+\left(\frac{\Delta S_{a l}}{S_{a l}}\right)^{2}}
$$

\section{Derivation of the standard deviation for Cassie contact angle, $\boldsymbol{\theta}_{C}^{*}$}

Cassie contact angle, $\theta_{C}^{*}$, from Eq. (4),

$$
\begin{aligned}
& \cos \theta_{C}^{*}=\frac{1}{D^{*}}\left(r_{\phi} \cos \theta-D^{*}+1\right) \\
& d \theta_{C}^{*}=\sqrt{\left(\frac{\partial \theta_{C}^{*}}{\partial \frac{1}{D^{*}}}\right)^{2}\left(d \frac{1}{D^{*}}\right)^{2}+\left(\frac{\partial \theta_{C}^{*}}{\partial r_{\phi}}\right)^{2} d r_{\phi}^{2}+\left(\frac{\partial \theta_{C}^{*}}{\partial \theta}\right)^{2} d \theta^{2}} \\
& \Delta \theta_{C}^{*}=\frac{180^{\circ}}{\pi} \sqrt{\frac{\left(r_{\phi} \cos \theta+1\right)^{2}\left(\Delta \frac{1}{D^{*}}\right)^{2}+\left(\frac{1}{D^{*}} \cos \theta \Delta r_{\phi}\right)^{2}+\left(\frac{\pi}{180^{\circ}} \frac{1}{D^{*}} r_{\phi} \sin \theta \Delta \theta\right)^{2}}{1-\left(\frac{1}{D^{*}} r_{\phi} \cos \theta-1+\frac{1}{D^{*}}\right)^{2}}}
\end{aligned}
$$

Here, $\Delta \frac{1}{D^{*}}$ in Eq. (S4) can be determined by replacing $D^{*}$ with $\frac{1}{D^{*}}$ in Eq. (S3). Eq. (S5) can also be used to calculate the standard deviation of $\theta_{C, s t a t}^{*}$ in Eq. (8) by replacing $2 R, 2(D+R), \frac{1}{D^{*}}$ and $\theta_{C}^{*}$ with $S_{a l}, R S_{m}$, $\frac{1}{D_{\text {stat }}^{*}}$ and $\theta_{C, \text { stat }}^{*}$, respectively. 


\section{Derivation of the standard deviation for robustness factor, $A^{*}$}

Robustness factor from Eq. (5), where we set $l_{c a p}=\sqrt{\gamma_{L V} / \rho g}$,

$A^{*}=\frac{2 \pi l_{\text {cap }}}{R\left(2 \sqrt{3} D^{*}-\pi\right)} \frac{1-\cos \theta}{\sqrt{D^{*}}-1+2 \sin \theta}$

$\frac{\partial A^{*}}{\partial \theta}=\frac{2 \pi l_{\text {cap }}}{R\left(2 \sqrt{3} D^{*}-\pi\right)} \frac{\left(\sqrt{D^{*}}-1+2 \sin \theta\right) \sin \theta-2(1-\cos \theta) \cos \theta}{\left(\sqrt{D^{*}}-1+2 \sin \theta\right)^{2}}=A_{1}$

$\frac{\partial A^{*}}{\partial S_{a l}}=-\frac{2 \pi l_{c a p}}{R^{2}\left(2 \sqrt{3} D^{*}-\pi\right)} \frac{1-\cos \theta}{\sqrt{D^{*}}-1+2 \sin \theta}=A_{2}$

$\frac{\partial A^{*}}{\partial D^{*}}=-\frac{2 \pi l_{c a p}}{R\left(2 \sqrt{3} D^{*}-\pi\right)} \frac{1-\cos \theta}{2 \sqrt{D^{*}}\left(\sqrt{D^{*}}-1+2 \sin \theta\right)^{2}}-\frac{1-\cos \theta}{\sqrt{D^{*}}-1+2 \sin \theta} \frac{4 \sqrt{3} \pi l_{c a p}}{R\left(2 \sqrt{3} D^{*}-\pi\right)^{2}}=A_{3}$

$d A^{*}=\sqrt{\left(\frac{\partial A^{*}}{\partial \theta}\right)^{2} d\left(\frac{\pi}{180^{\circ}} \theta\right)^{2}+\left(\frac{\partial A^{*}}{\partial R}\right)^{2} d R^{2}+\left(\frac{\partial A^{*}}{\partial D^{*}}\right)^{2} d D^{* 2}}$

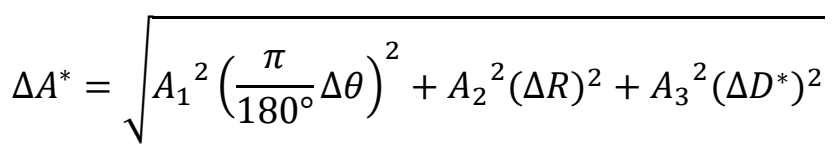

\section{Derivation of the standard deviation for statistical robustness factor, $\boldsymbol{A}_{\text {stat }}^{*}$}

Similarly, replacing $R, D^{*}$ and $A^{*}$ in Eq. (S6) with $S_{a l} / 2, D_{\text {stat }}^{*}$ and $A_{\text {stat }}^{*}$, the standard deviation of the statistical robustness factor, $A_{\text {stat }}^{*}$, can be determined as,

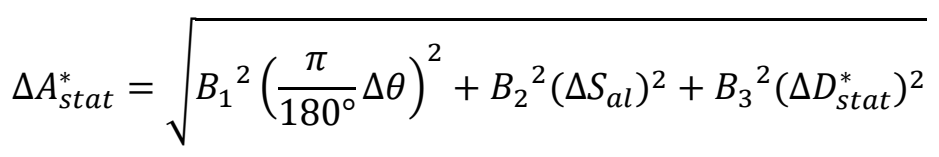

Where, $B_{1}=\frac{4 \pi l_{\text {cap }}}{S_{a l}\left(2 \sqrt{3} D_{\text {stat }}^{*}-\pi\right)} \frac{\left(\sqrt{D_{\text {stat }}^{*}}-1+2 \sin \theta\right) \sin \theta-(1-\cos ) \cos \theta}{\left(\sqrt{D_{\text {stat }}^{*}}-1+2 \sin \theta\right)^{2}}$,

$B_{2}=\frac{4 \pi l_{\text {cap }}}{S_{a l}\left(2 \sqrt{3} D_{\text {stat }}^{*}-\pi\right)} \frac{\left(\sqrt{D_{\text {stat }}^{*}}-1+2 \sin \theta\right) \sin \theta-2(1-\cos ) \cos \theta}{\left(\sqrt{D_{\text {stat }}^{*}}-1+2 \sin \theta\right)^{2}}$,

And, $B_{3}=-\frac{4 \pi l_{\text {cap }}}{S_{a l}\left(2 \sqrt{3} D_{\text {stat }}^{*}-\pi\right)} \frac{1-\cos }{2 \sqrt{D_{\text {stat }}^{*}}\left(\sqrt{D_{\text {stat }}^{*}}-1+2 \sin \theta\right)^{2}}-\frac{1-\cos \theta}{\sqrt{D_{\text {stat }}^{*}}-1+2 \sin \theta} \frac{4 \sqrt{3} \pi l_{\text {cap }}}{S_{\text {al }}\left(2 \sqrt{3} D_{\text {stat }}^{*}-\pi\right)^{2}}$

\section{Derivation of the standard deviation for critical contact angle, $\theta_{\text {crit }}$}

Critical equilibrium contact angle, $\theta_{\text {crit }}$, can be written in terms of feature size, $2 R$, and centre-to-centre feature spacing, $2(D+R)$, from Eq. (6) as, 


$$
\cos \theta_{c r i t}=\frac{[2 R]^{2}-[2(D+R)]^{2}}{\left(1+\frac{S_{d r}}{100}\right)[2(D+R)]^{2}-[2 R]^{2}}
$$

$$
\frac{\partial \theta_{\text {crit }}}{\partial[2 R]}=\frac{-1}{\sqrt{1-\left(\frac{[2 R]^{2}-[2(D+R)]^{2}}{\left(1+\frac{S_{d r}}{100}\right)[2(D+R)]^{2}-[2 R]^{2}}\right)^{2}}} \frac{0.02 S_{d r}[2 R][2(D+R)]^{2}}{\left(\left(1+\frac{S_{d r}}{100}\right)[2(D+R)]^{2}-[2 R]^{2}\right)^{2}}=C_{1}
$$

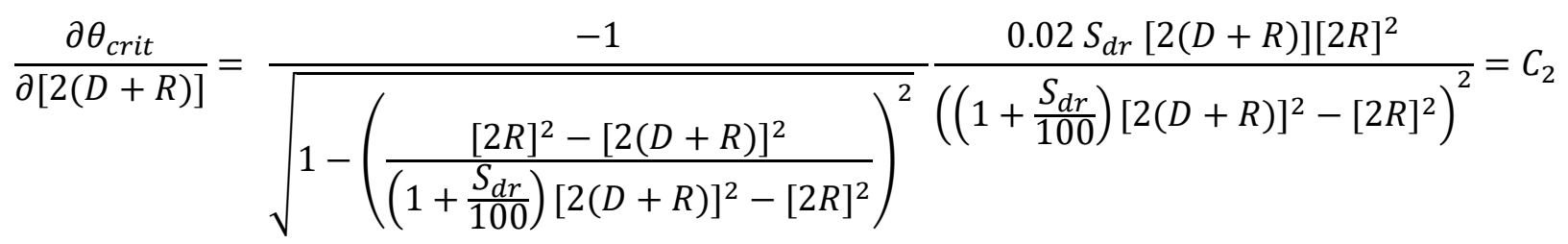

$\frac{\partial \theta_{c r i t}}{\partial S_{d r}}=\frac{1}{\sqrt{1-\left(\frac{[2 R]^{2}-[2(D+R)]^{2}}{\left(1+\frac{S_{d r}}{100}\right)[2(D+R)]^{2}-[2 R]^{2}}\right)^{2}}} \frac{0.01[2(D+R)]^{2}([2 R]-[2(D+R)])^{2}}{\left(\left(1+\frac{S_{d r}}{100}\right)[2(D+R)]^{2}-[2 R]^{2}\right)^{2}}=C_{3}$

$$
\begin{aligned}
& d \theta_{\text {crit }}=\sqrt{\left(\frac{\partial \theta_{\text {crit }}}{\partial[2 R]}\right)^{2} d[2 R]^{2}+\left(\frac{\partial \theta_{\text {crit }}}{\partial[2(D+R)]}\right)^{2} d[2(D+R)]^{2}+\left(\frac{\partial \theta_{\text {crit }}}{\partial S_{d r}}\right)^{2} d S_{d r}{ }^{2}}
\end{aligned}
$$

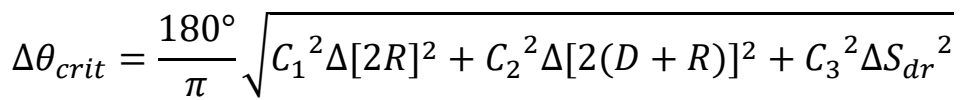

Eq. (S8) can also be used to calculate the standard deviation of $\theta_{\text {crit }}^{\text {stat }}$ in Eq. (10) by replacing $2 R, 2(D+$ $R$ ), and $\theta_{\text {crit }}$ with $S_{a l}, R S_{m}$, and $\theta_{\text {crit }}^{\text {stat }}$, respectively.

\section{Derivation of the standard deviation for the apparent contact angle in a mixed Cassie state, $\theta_{M}^{*}$}

From Eq. (11),

$\cos \theta_{M}^{*}=\phi_{W} \cos \theta_{W}^{*}+\left(1-\phi_{W}\right) \cos \theta_{C, \text { stat }}^{*}$

$\frac{\partial \theta_{M}^{*}}{\partial \theta_{W}^{*}}=\frac{\phi_{W} \sin \theta_{W}^{*}}{\sqrt{1-\left(\phi_{W} \cos \theta_{W}^{*}+\left(1-\phi_{W}\right) \cos \theta_{C, \text { stat }}^{*}\right)^{2}}}=D_{1}$

$\frac{\partial \theta_{M}^{*}}{\partial \theta_{C, \text { stat }}^{*}}=\frac{\left(1-\phi_{W}\right) \sin \theta_{C, \text { stat }}^{*}}{\sqrt{1-\left(\phi_{W} \cos \theta_{W}^{*}+\left(1-\phi_{W}\right) \cos \theta_{C, \text { stat }}^{*}\right)^{2}}}=D_{2}$

$d \theta_{M}^{*}=\frac{180^{\circ}}{\pi} \sqrt{\left(\frac{\partial \theta_{M}^{*}}{\partial \theta_{W}^{*}}\right)^{2} d\left(\frac{\pi \theta_{W}^{*}}{180^{\circ}}\right)^{2}+\left(\frac{\partial \theta_{M}^{*}}{\partial \theta_{C, \text { stat }}^{*}}\right)^{2} d\left(\frac{\pi \theta_{C, \text { stat }}^{*}}{180^{\circ}}\right)^{2}}$ 
$\Delta \theta_{M}^{*}=\frac{180^{\circ}}{\pi} \sqrt{\left(\frac{\pi D_{1} \Delta \theta_{W}^{*}}{180^{\circ}}\right)^{2}+\left(\frac{\pi D_{2} \Delta \theta_{C, s t a t}^{*}}{180^{\circ}}\right)^{2}}$

\section{References}

1. Coleman, H.W.; Steele, W.G. Experimentation, Validation, and Uncertainty Analysis for Engineers; John Wiley \& Sons, Inc.: New Jersey, 2009; p. 126. 